\title{
Unexpected Delayed Colon Perforation after the Endoscopic Submucosal Dissection with Snaring of a Laterally Spreading Tumor
}

\author{
Young Bo Ko, Jeong-Mi Lee, Wan Soo Kim, Min Seob Kwak, Ji Wan Lee, Dong Yeol Shin, Dong-Hoon Yang and Jeong-Sik Byeon \\ Department of Gastroenterology, Asan Medical Center, University of Ulsan College of Medicine, Seoul, Korea
}

Colonic perforation may occur as a complication of diagnostic and therapeutic colonoscopy. The risk factors for perforation after colorectal endoscopic submucosal dissection (ESD) include an inexperienced endoscopist, a large tumor size, and submucosal fibrosis. The mechanisms of perforation include unintended endoscopic resection/dissection and severe thermal injury. Here, we report a case of colon perforation that occurred after ESD with snaring of a laterally spreading tumor. The perforation was completely unexpected because there were no colorectal ESD-associated risk factors for perforation, deep dissection, or severe coagulation injury in our patient. Clin Endosc 2015;48:570-575

Key Words: Delayed perforation; Risk factor; Mechanism; Endoscopic submucosal dissection

\section{INTRODUCTION}

The number of complications associated with therapeutic colonoscopy has increased with the increasing number of these procedures. Of these complications, perforation is considered one of the most serious. The perforation rate during therapeutic colonoscopy ranges between $0.15 \%$ and $3 \%$. The incidence of perforation is even higher during endoscopic submucosal dissection (ESD) in the colorectum. ${ }^{1-3}$ The risk factors for colorectal ESD-associated perforation include inexperience on the part of the endoscopist (i.e., has performed $<50$ ESD procedures), a large tumor size, and submucosal fibrosis. ${ }^{1,4}$ Colonic perforation during colonoscopic polypectomy or colorectal ESD occurs as a result of unintended endoscopic resection/dissection and/or thermal injury. ${ }^{1}$ In the former case, immediate perforation predominantly occurs;

Received: April 3, 2015 Revised: May 11, 2015

Accepted: May 15, 2015

Correspondence: Jeong-Sik Byeon

Department of Gastroenterology, Asan Medical Center, University of Ulsan College of Medicine, 88 Olympic-ro 43-gil, Songpa-gu, Seoul 05505, Korea

Tel: +82-2-3010-3905, Fax: +82-2-476-0824, E-mail: jsbyeon@amc.seoul.kr

(cc) This is an Open Access article distributed under the terms of the Creative Commons Attribution Non-Commercial License (http://creativecommons.org/ licenses/by-nc/3.0) which permits unrestricted non-commercial use, distribution, and reproduction in any medium, provided the original work is properly cited. that is, colonic wall defects are seen during the procedure. However, in the case of thermal injury, the postprocedural ulcer base may demonstrate whitish coagulation damage, and delayed perforation mainly occurs.

We here report a case in which delayed perforation occurred after performing ESD on a laterally spreading tumor (LST) in the colon. This perforation was completely unexpected because there were no ESD-associated risk factors for this complication in the patient, and no severe coagulation damage was apparent at the post-ESD ulcer base. We discuss our findings for this patient and also review the relevant literature.

\section{CASE REPORT}

A 43-year-old man was referred to our center for the endoscopic resection of a nongranular LST (LST-NG) in the transverse colon. The histological diagnosis of the lesion was tubular adenoma with low-grade dysplasia. He had no relevant medical history. Colonoscopic resection was performed on hospital day 2. The colon was well prepared, and a flat, elevated LST-NG measuring $20 \times 22 \mathrm{~mm}$ was observed in the transverse colon. The lesion was type IV with a Kudo pit pattern, and clinically suggestive of noninvasive neoplasia. A submucosal cushion was created with a saline injection, and no 

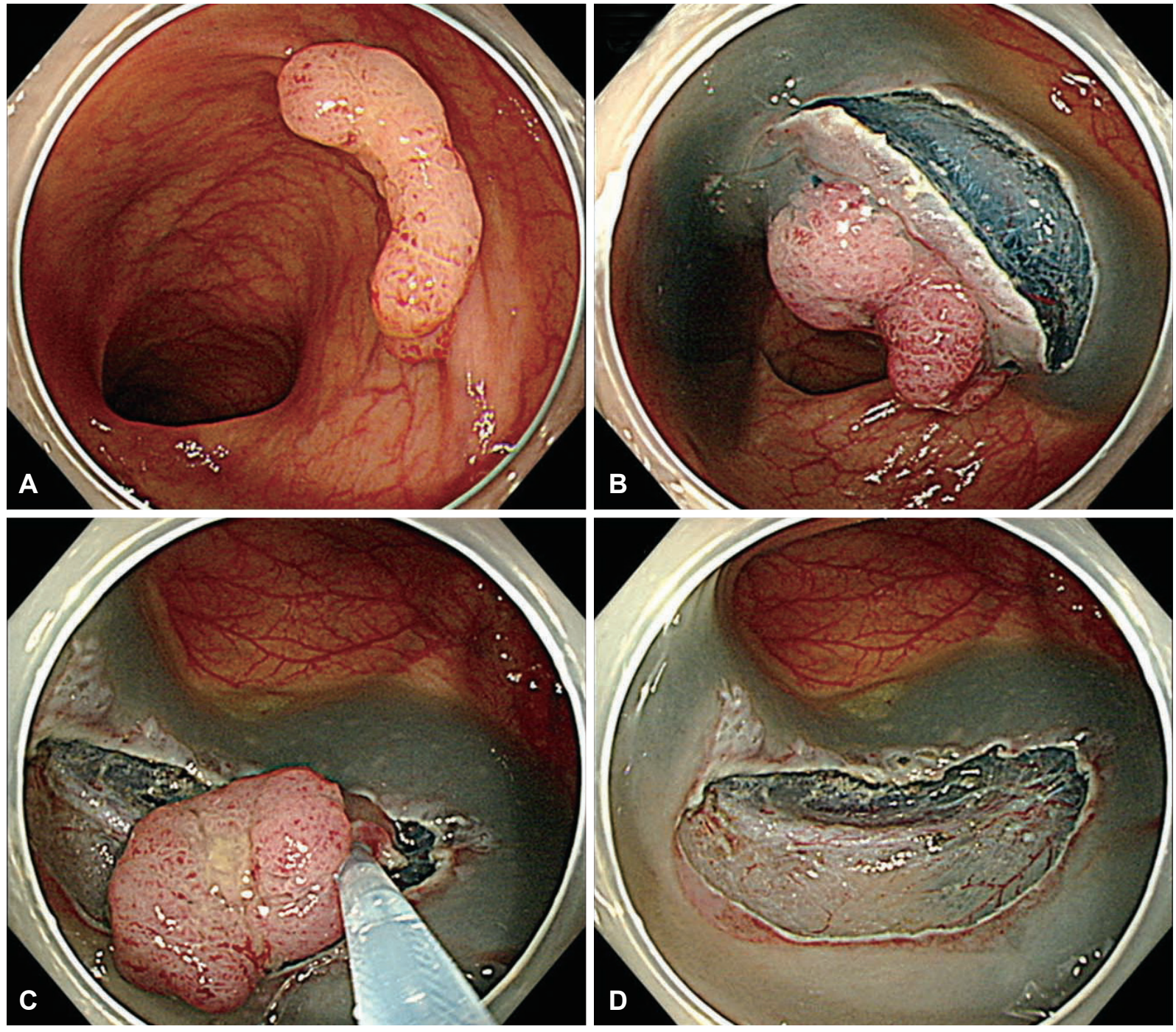

Fig. 1. (A) Nongranular laterally spreading tumor in the transverse colon. (B) Submucosal dissection was performed. (C) After partial endoscopic submucosal dissection (ESD), the lesion was removed en bloc by means of snaring. (D) En bloc resection was completed, and the post-ESD ulcer base was clean without bleeding, severe coagulation injury, or endoscopically evident perforation.

nonlifting sign or submucosal fibrosis was noted. Submucosal dissection was performed with a fixed flexsnare knife (Kachu Medico Co., Seoul, Korea). After the dissection of the lesion (except for the central portion), the residual attached portion was snared and removed en bloc. The duration of electrocauterization (i.e., the duration of submucosal dissection and snaring) was 23 minutes, and the total procedure time was 43 minutes. No barotraumas, deep thermal injuries, or other complications such as immediate bleeding or perforation were noted during the procedure. The post-ESD ulcer base was clean without bleeding, deep coagulation injury, or perforation (Fig. 1).

The patient had no abdominal pain and his vital signs were stable after colonoscopic resection. No free air was evident on postprocedural chest radiography or simple abdominal imaging. The following morning, however, he complained of mild abdominal discomfort. Sips of water were allowed, and he complained of severe abdominal pain several hours later. Abdominal tenderness was equivocal. Simple abdominal imaging was performed; however, no free gas was evident. Postpolypectomy electrocoagulation syndrome was tentatively diagnosed. His symptoms subsided after water intake was stopped. However, the same symptoms returned when sips of water were allowed a few hours later. Direct tenderness of the whole abdomen was noted on physical examination. His vital signs were stable; however, free air under the right diaphragm was noted on simple abdominal images (Fig. 2). His white blood cell count was $2800 / \mu \mathrm{L}$, and the C-reactive protein concentration was $5.85 \mathrm{mg} / \mathrm{dL}$. Abdominopelvic computed tomography was performed 30 hours after the procedure, which 

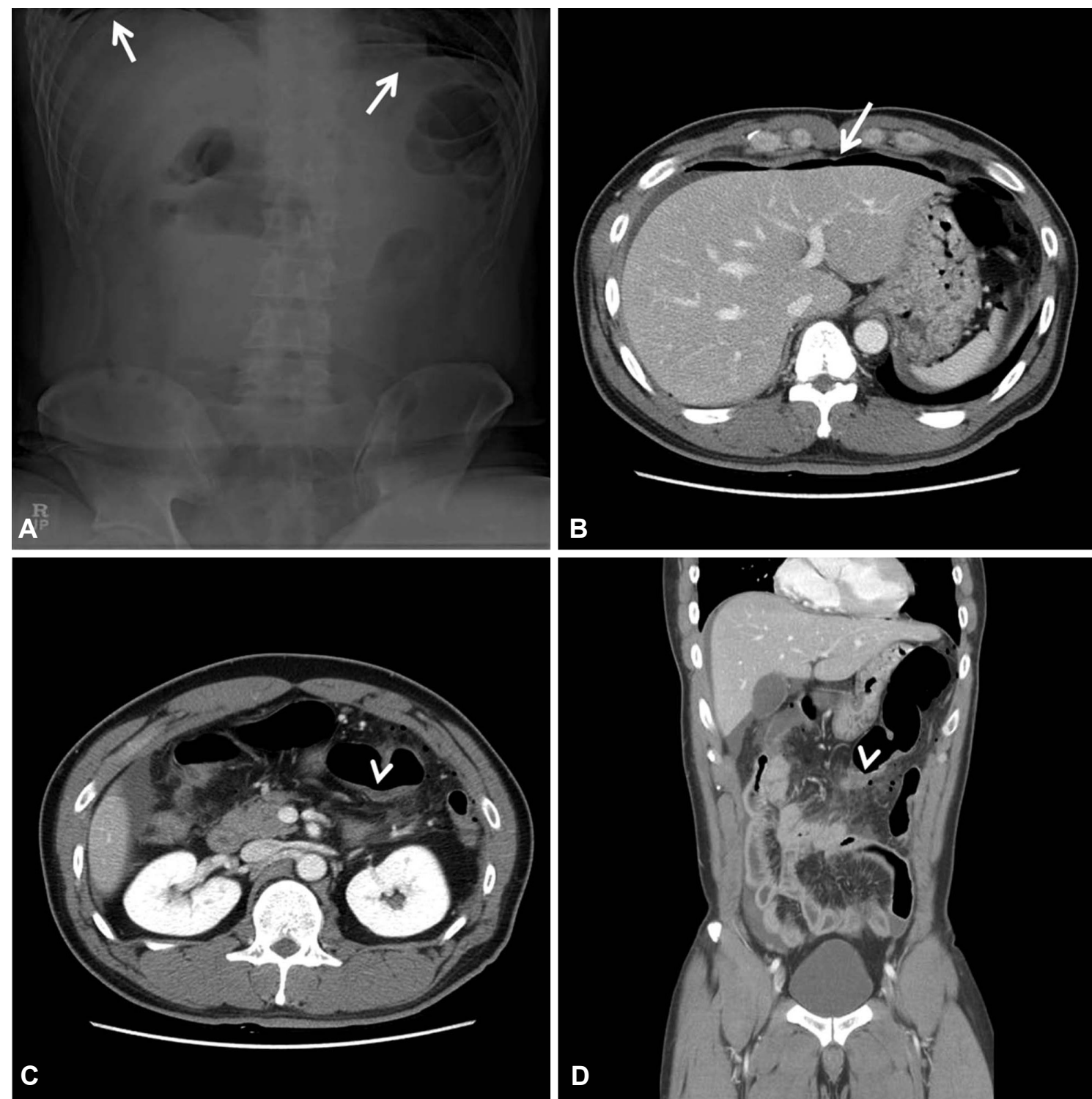

Fig. 2. (A, B) Pneumoperitoneum on simple abdominal imaging and abdominopelvic computed tomography (arrows). (C, D) Transverse colon wall thickening and irregularity (arrowheads) with pericolic free air.

revealed a pneumoperitoneum and wall irregularities at the endoscopic resection site (Fig. 2). Empirical antibiotics were administered, and an emergency laparoscopic operation was performed because the symptoms rapidly aggravated and the whole abdomen demonstrated rigidity with rebound tenderness, suggesting severe, aggravating peritonitis. The operative findings included severe peritoneal soiling and necrosis at the colonoscopic resection site. Primary repair was performed after massive irrigation of the soiled feces and removal of necrotic colon tissue.

The final pathologic diagnosis of the endoscopic resection specimen was mixed traditional serrated adenoma and tubular adenoma with clear resection margins. The depth of endoscopic resection reached the submucosal layer without affecting the proper muscle. The thermal injury to the submucosal layer was not severe (Fig. 3). The surgically resected colon specimen demonstrated wall necrosis, neutrophil infiltration, and a large hematoma in the adjacent proper muscle layer (Fig. 4). He was discharged after 7 days of postoperative care without any other adverse events.

\section{DISCUSSION}

Perforation is the most serious complication of diagnostic or therapeutic colonoscopy. Therapeutic colonoscopy demonstrates a higher perforation rate than does diagnostic colonoscopy $(0.15 \%$ to $3 \%)$, and the mortality rate can be as high as 

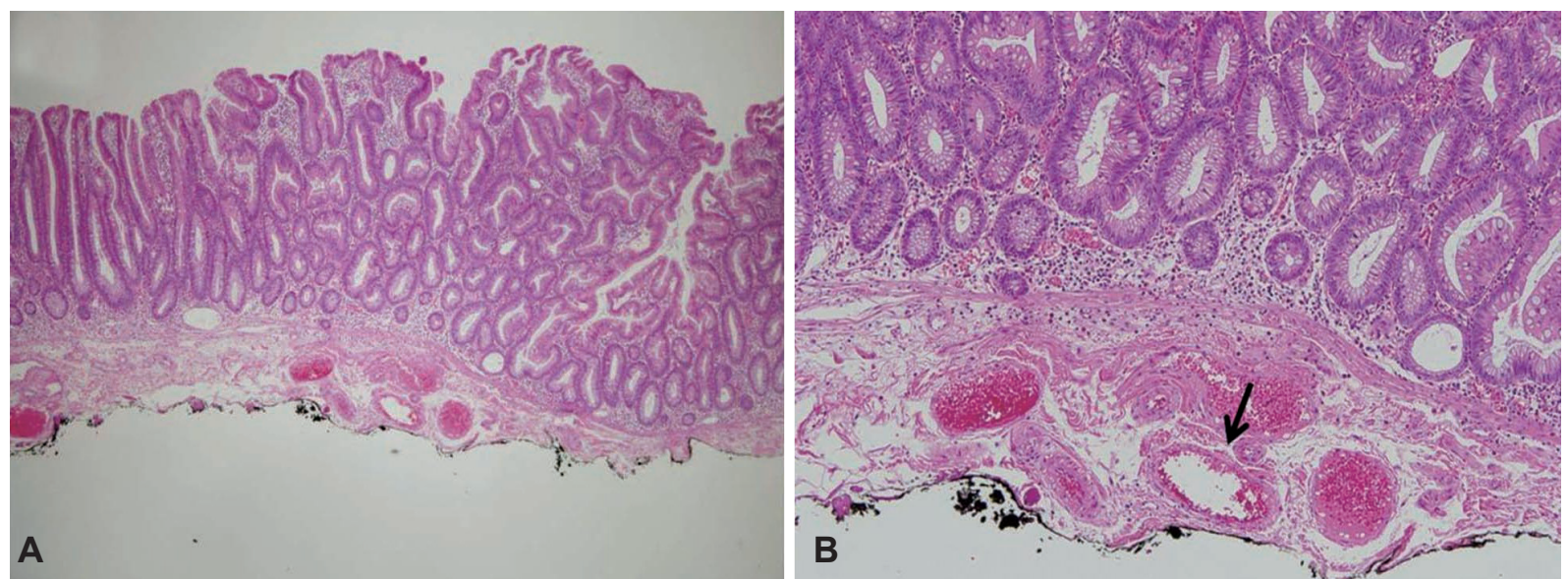

Fig. 3. Endoscopic resection specimen. (A) The specimen has mucosal and submucosal layers without the muscularis layer of the colonic wall (H\&E stain, $\times 40)$. (B) Submucosal layer showing submucosal vessels (arrow) without severe coagulation damage due to thermal injury (H\&E stain, $\times 100)$.
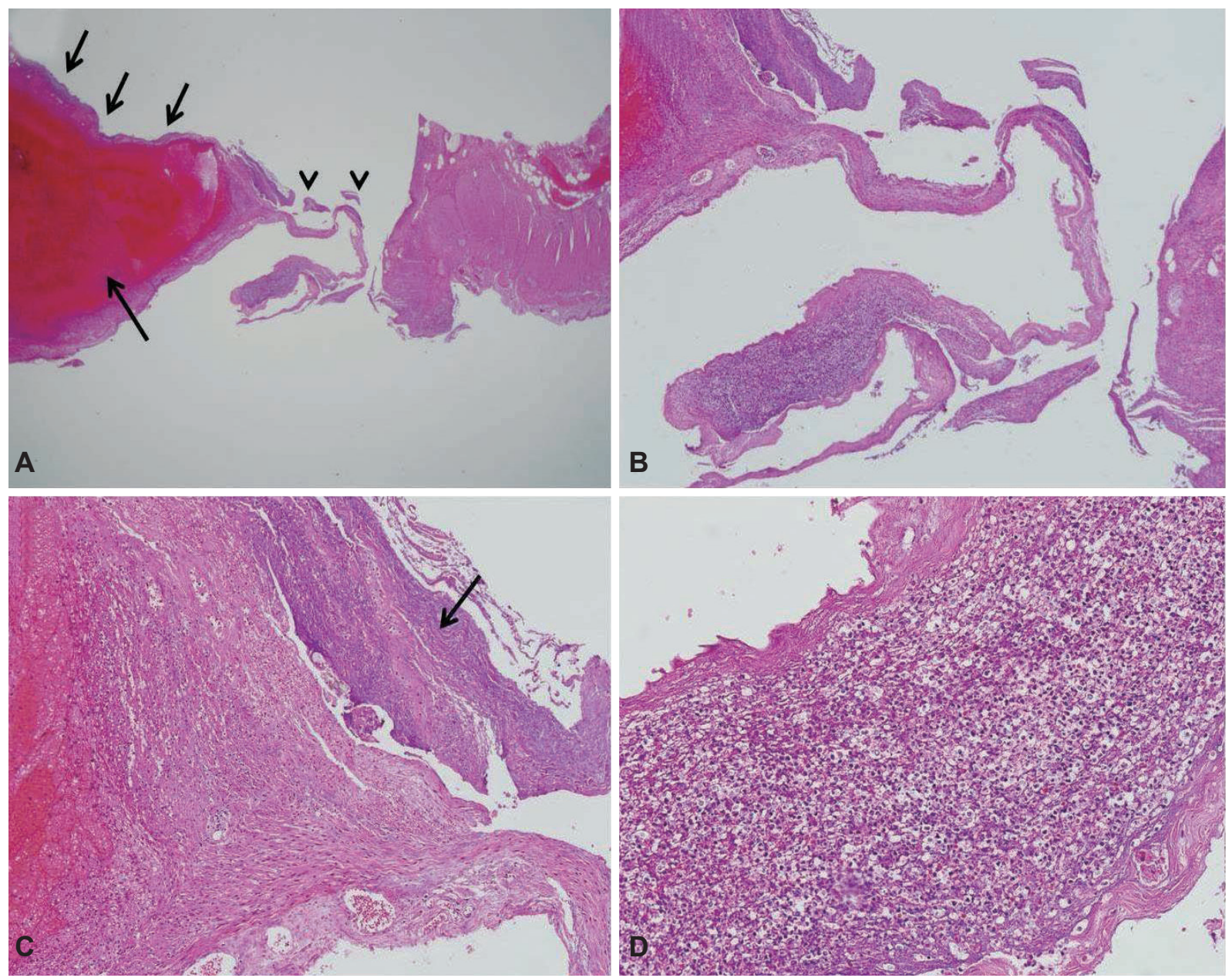

Fig. 4. Surgical resection specimen. (A) Large hematoma (long arrow) in the proper muscle layer. The endoscopic resection site (short arrows) and perforation site (arrowheads) are also shown (H\&E stain, $\times 12.5)$. (B) Perforation site in the specimen (H\&E stain, $\times 40)$. (C) Necrosis in the colonic wall is evident at the perforation site (arrow) (H\&E stain, $\times 100)$. (D) Neutrophil infiltration is evident at the perforation site (H\&E stain, $\times 200$ ).

$13 \%{ }^{1-3,5}$ Our current case demonstrated delayed perforation, which is not uncommon, accounting for $6 \%$ to $40 \%$ of colon perforations, mainly after therapeutic colonoscopies. ${ }^{5-7}$ Most notably, ESD, which was originally developed for the en bloc resection of large or ulcerative gastric epithelial neoplasms, demonstrates a high perforation rate ranging between 1.7\% 
and $20.4 \%{ }^{4,8-11}$ In our current case, endoscopic resection was performed by using ESD with snaring. ESD with snaring is a simplified alternative to ESD alone for the en bloc resection of large colorectal lesions. Although the procedure duration of ESD with snaring is usually shorter than that of ESD without snaring, the perforation risk associated with ESD with snaring was not significantly lower than that of ESD without snaring ( $3 \%$ vs. $7 \%, p=0.24$ ) in our previous study. ${ }^{12}$

Perforation can occur after colonoscopy and it is not a negligible complication, and therefore determining if the patient or the procedure has any risk factors for colon perforation would help promote safe clinical practice. The risk factors for colonoscopic perforation include female sex, comorbidities, older age ( $>75$ years), diverticulosis, bowel obstruction, and a history of intra-abdominal surgery. ${ }^{1,13}$ In the case of colorectal ESD, a large tumor size (i.e., $\geq 50 \mathrm{~mm}$ ), an inexperienced endoscopist, and submucosal fibrosis are well-known risk factors for perforation. ${ }^{4,10,11}$ However, none of these factors were present in our current case. In addition, during the procedure, there was neither a deep dissection that injured the proper muscle layer nor excessive thermal injury that resulted in severe coagulation damage to the submucosa and proper muscle. Furthermore, the endoscopic resection specimen did not reach the proper muscle layer or indicate severe tissue injury.

We suggest several possible explanations for the unexpected perforation in our case, on the basis of the histopathology of the surgical specimen. First, when considering a large hematoma in the proper muscle layer adjacent to the perforation site, a high-tension colonic wall might lead to perforation. However, perforation did not occur at the center of hematoma where the wall tension might be highest. Therefore, perforation cannot be explained solely by this hypothesis. A second possibility is that acute infection followed by massive inflammation and necrosis in the colonic wall may have occurred because there was severe neutrophil infiltration. However, neutrophil infiltration could arise after wall necrosis. Therefore, it is difficult to know the causal relation between neutrophil infiltration and necrosis. Third, delayed perforation may occur because of the excessive electrical coagulation of vessels in the submucosa and proper muscle layer. A long duration of electrocauterization may predispose vessels to coagulation injury, thereby resulting in delayed perforation. In our case, the cautery time was 23 minutes because ESD with snaring was performed. This cautery duration is longer than the average cautery time of conventional polypectomy, which is usually $<1$ minute. We suggest that this long electrocauterization time might have contributed to the delayed perforation. Thus, the perforation mechanism in our current case remains unclear, and we believe several mechanisms together may have contributed to this adverse event.
The treatments for perforation include surgical and nonsurgical management. In the past, surgery was the mainstay of treatment and medical management was only performed on select patients. ${ }^{1}$ However, as endoscopic clipping techniques have progressed, the need for and the frequency of surgery have decreased. Our group has reported the clinical courses of 38 perforations after both diagnostic $(n=13)$ and therapeutic $(n=25)$ colonoscopies. Of these cases, only nine patients (23\%) required surgery. Most other patients who had undergone colonoscopic clipping were treated with conservative management. ${ }^{14}$ However, patients who receive a nonsurgical intervention should receive close follow-up examinations because immediate surgery needs to be performed if clinical deterioration occurs. Surgery is indicated for patients with large perforations, generalized peritonitis, ongoing sepsis, and deterioration despite being managed conservatively. ${ }^{1}$ In our current case, rapid progressive abdominal pain with tenderness and rebound tenderness developed in the whole abdomen, indicating severe, generalized peritonitis. Therefore, emergency surgery was performed rather than endoscopic clipping. Severe peritoneal soiling with large necrotic tissue at the endoscopic resection site was noted in the surgical field. We believe that endoscopic clipping may have resulted in the adverse outcome in our case because we could not clean the soiled content, and successful clipping may have been prevented by the necrotic wall. Thus, we believe the decision to perform emergency surgery was appropriate.

Therapeutic colonoscopy, which has dramatically evolved in recent years, is an important modality for the treatment of colonic neoplasm. However, as shown in our current case, unexpected critical complications such as perforation can develop. Endoscopists should always keep in mind that perforation can develop even after clean procedures, and should closely evaluate any complaints about unexplained abdominal pain after therapeutic colonoscopy procedures, including polypectomy and ESD.

\section{Conflicts of Interest}

The authors have no financial conflicts of interest.

\section{REFERENCES}

1. Raju GS, Saito Y, Matsuda T, Kaltenbach T, Soetikno R. Endoscopic management of colonoscopic perforations (with videos). Gastrointest Endosc 2011;74:1380-1388.

2. Panteris V, Haringsma J, Kuipers EJ. Colonoscopy perforation rate, mechanisms and outcome: from diagnostic to therapeutic colonoscopy. Endoscopy 2009;41:941-951.

3. Mizushima T, Kato M, Iwanaga I, et al. Technical difficulty according to location, and risk factors for perforation, in endoscopic submucosal dissection of colorectal tumors. Surg Endosc 2015;29:133-139.

4. Isomoto $\mathrm{H}$, Nishiyama $\mathrm{H}$, Yamaguchi $\mathrm{N}$, et al. Clinicopathological fac- 
tors associated with clinical outcomes of endoscopic submucosal dissection for colorectal epithelial neoplasms. Endoscopy 2009;41:679-683.

5. Xiao YF, Bai JY, Yu J, et al. Endoscopic treatment of delayed colon perforation: the enteroscopy overtube approach. Endoscopy 2014;46:503508.

6. Hagel AF, Boxberger F, Dauth W, Kessler HP, Neurath MF, Raithel M. Colonoscopy-associated perforation: a 7-year survey of in-hospital frequency, treatment and outcome in a German university hospital. Colorectal Dis 2012;14:1121-1125.

7. Araghizadeh FY, Timmcke AE, Opelka FG, Hicks TC, Beck DE. Colonoscopic perforations. Dis Colon Rectum 2001;44:713-716.

8. Fujishiro M, Yahagi N, Kakushima N, et al. Outcomes of endoscopic submucosal dissection for colorectal epithelial neoplasms in 200 consecutive cases. Clin Gastroenterol Hepatol 2007;5:678-683.

9. Nakamura F, Saito Y, Sakamoto T, et al. Potential perioperative advantage of colorectal endoscopic submucosal dissection versus laparosco- py-assisted colectomy. Surg Endosc 2015;29:596-606.

10. Saito Y, Uraoka T, Yamaguchi Y, et al. A prospective, multicenter study of 1111 colorectal endoscopic submucosal dissections (with video). Gastrointest Endosc 2010;72:1217-1225.

11. Kim ES, Cho KB, Park KS, et al. Factors predictive of perforation during endoscopic submucosal dissection for the treatment of colorectal tumors. Endoscopy 2011;43:573-578.

12. Byeon JS, Yang DH, Kim KJ, et al. Endoscopic submucosal dissection with or without snaring for colorectal neoplasms. Gastrointest Endosc 2011;74:1075-1083.

13. Lohsiriwat V. Colonoscopic perforation: incidence, risk factors, management and outcome. World J Gastroenterol 2010;16:425-430.

14. Yang DH, Byeon JS, Lee KH, et al. Is endoscopic closure with clips effective for both diagnostic and therapeutic colonoscopy-associated bowel perforation? Surg Endosc 2010;24:1177-1185. 